\title{
Optimización multiobjetivo del proceso de soldeo GMAW de la aleación AA 6063-T5 basado en la penetración y en la zona afectada térmicamente
}

\author{
Valentín Miguel $^{\mathrm{a}, \mathrm{b}, \bowtie}$, Fernando Marín-Ortiz $^{\mathrm{a}}$, María C. Manjabacas ${ }^{\mathrm{a}, \mathrm{b}}$, \\ Eusebio J. Martínez-Conesa ${ }^{\mathrm{c}}$, Alberto Martínez-Martínez ${ }^{\mathrm{b}}$, Juana Coello ${ }^{\mathrm{a}, \mathrm{b}}$ \\ ${ }^{a}$ Escuela de Ingenieros Industriales de Albacete (EIIAB), UCLM. Avenida de España s/n, Campus Universitario, 02071 Albacete, España \\ ${ }^{\text {b}}$ Laboratorio de Ciencia e Ingeniería de Materiales (CIMA), Instituto de Desarrollo Regional (IDR). \\ Universidad de Castilla-La Mancha, 02071 Albacete, España \\ 'Departamento de Tecnología de Edificación, Universidad Politécnica de Cartagena (UPCT), \\ Pza. del Cronista Isidoro Valverde, Edif. La Milagrosa, 30202 Cartagena, España \\ ${ }^{\bowtie}$ Autor para la correspondencia: valentin.miguel@uclm.es
}

Enviado: 22 Julio 2014; Aceptado: 26 Enero 2015; Publicado on-Line: 23 Febrero 2015

\begin{abstract}
RESUMEN: La selección de los parámetros tecnológicos que regulan los procesos de soldadura por arco debe efectuarse de forma que se optimice el resultado de la operación. La penetración es uno de los factores decisivos, ya que es determinante en el modo de ejecutar el proceso así como en el comportamiento de la unión. Otro factor importante es la Zona Afectada Térmicamente (ZAT), cuando ésta presenta propiedades diferentes a las del material base. El modo en que afectan los parámetros tecnológicos en ambos, penetración y ZAT, es inverso a los objetivos que suelen plantearse. En este trabajo se presenta una metodología de optimización del proceso GMAW de la aleación AA 6063-T5 basado en el diseño de experimentos y la superficie de respuesta. Los resultados obtenidos permiten evaluar la conveniencia de fijar como objetivo valores de penetración tendentes a mantener ZAT en ratios asumibles. Se obtiene que la variable que más afecta a la optimización es la velocidad de soldadura.
\end{abstract}

PALABRAS CLAVE: Aluminio; GMAW; Metodología de Superficie de Respuesta (MSR); Optimización

Citation / Cómo citar este artículo: Miguel, V., Marín-Ortiz, F., Manjabacas, M.C., Martínez-Conesa, E.J., MartínezMartínez, A., Coello, J. (2015) "Optimización multiobjetivo del proceso de soldeo GMAW de la aleación AA 6063-T5 basado en la penetración y en la zona afectada térmicamente". Rev. Metal. 51(1): e037. doi: http://dx.doi.org/10.3989/ revmetalm.037.

ABSTRACT: Multiobjective optimization of GMAW process of the AA 6063-t5 alloy based on penetration and heat affected zone. The selection of technological parameters in a welding process must be led to the optimized results of the operation. Penetration is one of the most decisive factors for the success of the joint. Another important factor is the Heat Affected Zone (HAZ), when the mechanical properties of this one are modified respecting to the base material. The way in which the technological variables of the process affects to both them, penetration and HAZ, are inverse in each case. This work presents an optimization methodology based on the Design of Experiments (DOE) and the Response Surface Method (RSM) of the GMAW process applied to the aluminum alloy AA 6063-T5. This research is focused to obtain penetration rates that make HAZ values to be suitable in current applications. Welding rate has been found to be the most significant technological parameter for controlling the process.

KEYWORDS: Aluminum; GMAW; Optimization; Response Surface Methodology (RSM)

Copyright: (C) 2015 CSIC. This is an open-access article distributed under the terms of the Creative Commons Attribution-Non Commercial (by-nc) Spain 3.0 License. 


\section{INTRODUCCIÓN}

Las propiedades mecánicas de una unión soldada dependen de la geometría del cordón de soldadura, entre otros factores. El estudio de los factores geométricos del cordón de soldadura tiene una consideración importante para el diseño y fabricación de construcciones soldadas (Miguel et al., 2012). Uno de los parámetros más importantes es la penetración, que se emplea para describir la situación en que el metal depositado y el metal base no se funden de forma integral en la raíz de la soldadura, siempre que estuviera proyectado de este modo. La falta de penetración puede ser ocasionada porque la cara de la raíz de la soldadura no alcance la temperatura de fusión en toda su altura, porque el metal de aportación no llegue a la raíz o también puede deberse a la falta de disolución de los óxidos e impurezas de la superficie que impiden la unión. No obstante, las dos últimas causas pueden evitarse tomando la precaución de hacer una correcta preparación de bordes y de limpiar las superficies a unir, respectivamente. Las adecuadas condiciones térmicas de la unión hay que abordarlas desde el punto de vista de la investigación de los parámetros que afectan al arco eléctrico con el fin de controlar la penetración incompleta de la unión, que no es deseable, particularmente si la raíz de la soldadura está sometida a solicitaciones. Generalmente se obtiene una elevada penetración cuando el calor aportado térmicamente en el proceso es alto, estableciéndose cierto paralelismo entre ambos términos. La separación entre bordes y la velocidad de alimentación del electrodo son los parámetros más influyentes. Por otra parte, no se puede establecer una relación directa entre los parámetros geométricos de la unión respecto de la penetración (Miguel et al., 2012).

Otro factor importante a tener en cuenta en una unión soldada es la zona afectada térmicamente, definida como la longitud de material base cuyas propiedades se han visto modificadas por el proceso, generalmente como consecuencia de cambios microestructurales en el estado de partida del material. Aunque pueden establecerse objetivos diferentes para la ZAT (Zona afectada térmicamente) (Martínez et al., 2011) generalmente es deseable la minimización de dicha zona. Esto suele requerir criterios inversos en la selección de parámetros de soldadura respecto a lo indicado para la penetración. Efectivamente, en términos generales se puede afirmar que la minimización de la zona afectada térmicamente se consigue con una selección de parámetros tendentes a mantener el calor aportado en el nivel más bajo posible.

La selección de parámetros de soldeo también debe hacerse dentro de los márgenes en los que se consigue la estabilidad del punto de funcionamiento del arco eléctrico, por lo que la optimización del proceso debe hacerse en dicho rango (Miguel et al., 2009).
Diversos autores analizan la relación existente entre los parámetros del proceso de soldeo con la geometría de los cordones obtenidos planteando modelos en los que las funciones de respuesta, $Y$, se expresan conforme al modelo propuesto por McGlone y Chadwick (1978) en función de las variables del proceso, $X_{1}$ a $X_{n}$, tal y como se indica en la ecuación (1) en la que $b_{1} a b_{n}$ son las constantes de ajuste del modelo que dependen de los parámetros empleados en el proceso de soldeo.

$Y=K X_{1}^{b_{1}} X_{2}^{b_{2}} \cdots X_{n}^{b_{n}}$

Los valores de los coeficientes $b_{1} \ldots b_{n}$ son calculados mediante regresión múltiple. Karadeniz et al. (2007), determinan un modelo tipo McGlone y Chadwick también denominado ajuste curvilíneo, considerando como variables de influencia la tensión e intensidad del arco eléctrico y la velocidad de soldeo. Consideran únicamente el estudio de la penetración por soldadura MAG (Metal Active Gas) en aceros de bajo contenido en carbono y se limitan a obtener la variación de dicho parámetro en función de las variables indicadas. Wahab y Painter (1997) consideran como variables del proceso la tensión e intensidad del arco, la velocidad de soldeo y el caudal de gas empleado, limitándose a obtener un modelo similar al indicado. Kim et al. (2001) también se basan en el modelo curvilíneo, obteniendo como funciones de respuesta la penetración, el sobreespesor y la anchura del cordón. Estos autores establecen que la exactitud de los modelos encontrados varía desde 0 a $25 \%$. En todos los trabajos mencionados hasta el momento, existe como denominador común no solo el modelo empleado sino también que no se establece ninguna metodología de optimización con las variables de respuesta.

Kim et al. (2003), correlacionan la penetración del cordón de soldadura con la intensidad, tensión, velocidad y ángulo de soldeo. Comparan los resultados obtenidos mediante el modelo curvilíneo con los que se obtienen de una correlación lineal con las variables experimentadas. Los autores demuestran que se comporta mejor el modelo lineal. Concretamente, si se analizan los resultados obtenidos por estos autores, se puede estimar que el error medio cometido en una regresión lineal es del 16\% en tanto que el modelo curvilíneo que representa la ecuación (1) conduce a un error medio del 23\%. Ambos métodos presentan algún valor experimental que se aleja en más del $50 \%$ del correspondiente modelo teórico. Existen autores que emplean correlaciones de la geometría del cordón con el valor de energía aportada en el proceso. Murray y Scotti (1999) demuestran que la profundidad del cordón de soldadura en procesos GMAW (Gas Metal Arc Welding) de acero inoxidable depende del modo de transferencia del electrodo al metal, estableciendo una correlación en función de los parámetros 
transferencia de masa y transferencia de calor adimensionales. La correlación encontrada permite determinar la profundidad del cordón para diferentes modos de transferencia de material. Dorta et al. (2011) obtienen valores de la geometría del cordón para soldadura de aceros inoxidables dúplex en función de la tensión, la velocidad de soldeo y de la intensidad de corriente. También evalúan la correlación existente entre la geometría y la energía aportada. De este modo cuantifican la tensión, intensidad de corriente y la velocidad de soldeo en una sola variable, obteniendo índices de correlación significativos. Ello permite establecer que los efectos de una de las variables no son contrarrestados por las otras. Las funciones de correlación obtenidas son de tipo curvilíneo o polinómico.

Payares et al. (1997) determinan el área del cordón de soldadura en función de la tensión, intensidad y velocidad de soldeo en soldaduras a tope de la aleación de aluminio 6063-T5. Obtienen funciones de tipo curvilíneo para el área transversal estimada del cordón de soldadura con los parámetros tecnológicos del proceso.

No existen investigaciones dirigidas a la optimización de los diversos parámetros tecnológicos que intervienen en el proceso conjuntamente con el objetivo de controlar la penetración y la ZAT simultáneamente, estableciéndose simplificaciones en la ejecución de cordones de soldadura que no tienen en cuenta, por ejemplo, la separación entre las superficies a unir, cuando es sabido que este parámetro es determinante en los procesos de soldeo a tope con y sin preparación de bordes. Miguel et al. (2012) estudian la optimización de la geometría del cordón para la aleación de aluminio 6063-T5 considerando como variables tecnológicas la separación de bordes, la velocidad de soldeo, la tensión y la velocidad de alimentación del electrodo en un proceso GMAW automatizado. Aunque se hace alguna referencia a la ZAT, no se mide experimentalmente. El procedimiento empleado por dichos autores para la medición de la penetración es mejorable mediante técnicas metalográficas, lo que permitiría aumentar el grado de concordancia del modelo penetración-variables tecnológicas con los resultados experimentales.

En el presente trabajo, se lleva a cabo la modelización del proceso de soldeo de la aleación de aluminio 6063-T5 mediante la metodología de superficie de respuesta para soldaduras a tope con diferentes grados de separación entre superficies, considerando como variables de respuesta la penetración y la zona afectada térmicamente. A partir del modelo obtenido se optimiza el proceso obteniendo diferentes valores optimizados para las variables de respuesta para un objetivo de penetración establecido, así como los mínimos valores de la ZAT compatibles con los objetivos planteados. De este modo, se propone una metodología de optimización de procesos
GMAW aplicados a la aleación AA 6063-T5 basados en la superficie de respuesta y en la optimización de la penetración y de la ZAT en los cordones efectuados.

\section{MATERIALES Y MÉTODOS}

El material utilizado en los ensayos es la aleación de aluminio AA 6063-T5. Se llevaron a cabo cupones de soldadura de dimensiones $100 \times 40 \times 8 \mathrm{~mm}^{3}$. El procedimiento de soldeo empleado fue GMAW empleando argón puro comercial al 99\% típico de estas aplicaciones y se seleccionó un caudal de $141 \mathrm{~min}^{-1}$, suficiente para garantizar la protección del proceso (Miguel et al., 2009). La distancia entre la antorcha y la superficie de soldeo se mantuvo en $3 \mathrm{~mm}$ para garantizar la protección del gas. El electrodo empleado corresponde a la denominación S Al $5356\left(\mathrm{AlMg}_{5} \mathrm{Cr}(\mathrm{A})\right)$ conforme a la norma EN ISO 18273 (2005), de 1,2 mm de diámetro, típico de aplicaciones generales. El equipo de soldadura empleado fue el modelo TransPuls Synergic 2700 de la marca FRONIUS. Para la sujeción correcta de las probetas se realizó un soporte de aluminio que se alojó en un robot pórtico y cuyo objetivo era la colocación de los cupones a soldar y su fijación mediante pernos. Antes del proceso de soldeo, las zonas a unir fueron tratadas superficialmente con un cepillo de púas de aluminio para eliminar cualquier tipo de oxidación superficial que dificultara la estabilidad del arco eléctrico durante la soldadura. Para controlar la separación entre las piezas, variable en el diseño de experimentos, se emplearon chapas de $1 \mathrm{y}$ $2 \mathrm{~mm}$ de espesor, de forma que se ajustaba la colocación de las piezas a soldar en el utillaje mediante intercalado de las chapas mencionadas. Los parámetros de tensión y velocidad de alimentación del electrodo fueron regulados mediante los controles existentes en la equipo de soldadura. La velocidad de soldeo se estableció mediante programación por control numérico del movimiento del robot pórtico (Miguel et al., 2012). Los parámetros empleados se indican en la Tabla 1.

La metodología de la superficie de respuesta se aplicó adoptando un modelo cuadrático, conforme a lo indicado en la ecuación (2), en la que los coeficientes $\beta_{\mathrm{i}}$ corresponden a los coeficientes de regresión característicos y las variables de entrada de la función se corresponden con los valores $X_{i}, X_{j}$. El valor del residuo, $\varepsilon$, corresponde a la diferencia del valor experimental y del obtenido con la función para la variable de salida $Y$.

$$
Y=\beta_{0}+\sum_{i=1}^{k} \beta_{i} X_{i}+\sum_{i=1}^{k} \beta_{i i} X_{i}^{2}+\sum_{j=2} \sum_{i=1}^{k} \beta_{i j} X_{i} X_{j}+\varepsilon
$$

Se desarrolló un diseño de experimentos conforme a los valores de las variables de entrada indicados en la Tabla 1. El intervalo de valores seleccionados para las variables de soldadura 
corresponde a valores reales utilizados en los procedimientos típicos de soldeo de estas aleaciones.

La evaluación de la zona afectada térmicamente se llevó a cabo caracterizando la unión soldada mediante microscopía óptica y mediante barridos de microdureza HV 0,3 conforme a lo establecido en la norma UNE-EN ISO 6507-1 (2006). El equipo empleado en las medidas de microdureza fue un Shimadzu-2, determinando el valor de las mismas mediante el procesado asistido del equipo. Los barridos de microdureza se realizaron a partir del centro del cordón de soldadura con $1 \mathrm{~mm}$ de separación y tomando como directriz de referencia la mitad de la penetración. La penetración se ha medido mediante el empleo de una lupa binocular y un análisis de imagen sobre los cordones perfectamente pulidos con paños lubricados con etilenglicol y pasta de diamante de granulometría $1 \mu \mathrm{m}$ (Fig. 1). Este método permite definir mucho mejor la penetración real del cordón que el método empleado en otras investigaciones (Miguel et al., 2012).

TABla 1. Parámetros de los experimentos realizados (DOE) (Miguel et al., 2012)

\begin{tabular}{|c|c|c|c|c|}
\hline Exp. & S (mm) & $\mathrm{Vs}\left(\mathrm{mm} \mathrm{s}^{-1}\right)$ & $\mathrm{T}(\mathrm{V})$ & $\mathrm{Ve}\left(\mathrm{m} \mathrm{min}^{-1}\right)$ \\
\hline 1 & 1 & 13 & 18 & 12 \\
\hline 2 & 1 & 13 & 24 & 12 \\
\hline 3 & 2 & 13 & 24 & 10,5 \\
\hline 4 & 2 & 13 & 21 & 12 \\
\hline 5 & 2 & 17,8 & 21 & 10,5 \\
\hline 6 & 1 & 17,8 & 24 & 10,5 \\
\hline 7 & 0 & 13 & 18 & 10,5 \\
\hline 8 & 0 & 8,2 & 21 & 10,5 \\
\hline 9 & 1 & 13 & 18 & 9 \\
\hline 10 & 2 & 13 & 18 & 10,5 \\
\hline 11 & 0 & 13 & 21 & 12 \\
\hline 12 & 0 & 13 & 24 & 10,5 \\
\hline 13 & 0 & 13 & 21 & 9 \\
\hline 14 & 1 & 17,8 & 18 & 10,5 \\
\hline 15 & 0 & 17,8 & 21 & 10,5 \\
\hline 16 & 1 & 8,2 & 21 & 12 \\
\hline 17 & 2 & 8,2 & 21 & 10,5 \\
\hline 18 & 1 & 17,8 & 21 & 12 \\
\hline 19 & 1 & 13 & 24 & 9 \\
\hline 20 & 1 & 8,2 & 18 & 10,5 \\
\hline 21 & 1 & 8,2 & 21 & 9 \\
\hline 22 & 1 & 8,2 & 24 & 10,5 \\
\hline 23 & 1 & 17,8 & 21 & 9 \\
\hline 24 & 2 & 13 & 21 & 9 \\
\hline
\end{tabular}

S: Separación; Vs: Velocidad de Soldadura; T: Tensión; Ve: Velocidad Electrodo.

\section{RESULTADOS Y DISCUSIÓN}

Los resultados de dureza en función de la distancia al centro del cordón de soldadura para los diferentes experimentos permitieron establecer claramente el valor de la ZAT. Como una de las variables consideradas en el experimento es la separación de los bordes a unir, se ha restado dicha distancia en la expresión de los resultados para hacerlos comparables. En la Tabla 2 se establecen los resultados obtenidos para la penetración y para la ZAT. El Método de la Superficie de Respuesta (MSR), se plantea de forma matricial conforme a lo indicado en la ecuación (3).

$[Y]=[X][B]$

La resolución de la ecuación (3) para la obtención de los coeficientes correspondientes a la modelización cuadrática de la superficie de respuesta se ha efectuado mediante la aplicación informática Minitab $16^{\circledR}$. Los resultados para las variables penetración, P, y ZAT se indican en las ecuaciones (4) y (5), respectivamente.

$\mathbf{P}(\mathrm{mm})=-23,2762+1,82156 \mathrm{~S}-0,313520 \mathbf{V}_{\mathrm{s}}$ $+2,24729 \mathbf{T}+0,373333 \mathbf{V}_{\mathrm{e}}-0,345 \mathbf{S}^{2}-0,00352648 \mathbf{V}_{\mathbf{s}}^{\frac{\mathrm{s}}{2}}$ $-0,0573611 \mathbf{T}^{2}+0.0130208 \mathbf{S V}_{\mathbf{s}}+0,0483333 \mathbf{S T}$ $-0,171667 \mathbf{S} \mathbf{V}_{\mathrm{s}}+0,00659722 \mathbf{V}_{\mathrm{s}} \mathbf{T}+0,0166667 \mathbf{V}_{\mathrm{s}} \mathbf{V}_{\mathrm{e}}$ $+0,00277778 \mathbf{T} \boldsymbol{V}_{\mathrm{e}}$

$\mathbf{Z A T}(\mathrm{mm})=-58,788+5,23958 \mathbf{S}+0,518663 \boldsymbol{V}_{s}$ $+4,49653 \mathrm{~T}+1,56944 \boldsymbol{V}_{e}+0,625 \boldsymbol{S}^{2}+0,0922309 \boldsymbol{V}_{s}^{\frac{S}{2}}$ $-0,0555556 \boldsymbol{T}^{2}-0,364583 \boldsymbol{S} \boldsymbol{V}_{s}-0,364583 \boldsymbol{S} \boldsymbol{V}_{S}$ $-1,54512 \cdot 10^{-17} \boldsymbol{S} \boldsymbol{T}-0,166667 \boldsymbol{S} \boldsymbol{V}_{e}$ $-0,121528 \boldsymbol{V}_{s} \boldsymbol{T}-0,0964444 \boldsymbol{V}_{s} \boldsymbol{V}_{e}-7,52151 \cdot 10^{-17} \boldsymbol{T} \boldsymbol{V}_{e}$

En las Figuras 2 y 3 se puede observar las gráficas de las superficies de respuesta para ambas variables en función de las variables tecnológicas del proceso de soldeo.

El análisis ANOVA de la penetración y de la ZAT permitió establecer un p-valor de $0,018 \mathrm{y}$ 0,000 , respectivamente, inferior a 0,05 , lo que significa que para un nivel de confianza establecido del 95\% ambas variables dependen al menos de una de las variables de entrada, es decir existe dependencia de la variable de salida respecto de las variables de entrada.

En la Figura 4 se representan las gráficas de los efectos principales de las variables tecnológicas. Los puntos de dichas gráficas son las medias de la variable de respuesta en los diversos niveles de cada factor. Lógicamente se observa que los efectos de las variables tecnológicas son similares para cada una de las variables de salida, habida cuenta de que un mayor calor aportado en el proceso conduce a una mayor penetración y a una mayor ZAT. La separación de las piezas a soldar no ejerce influencia en 
TABLA 2. Resultados de la penetración y de la ZAT: valores experimentales vs valores modelizados mediante MSR

\begin{tabular}{|c|c|c|c|c|}
\hline Experimento & $\begin{array}{c}\text { Penetración } \\
\text { Experimental P (mm) }\end{array}$ & $\begin{array}{l}\text { Penetración } \\
\text { MSR P }(\mathbf{m m})\end{array}$ & $\begin{array}{c}\text { ZAT Experimental } \\
(\mathrm{mm})\end{array}$ & $\begin{array}{c}\text { ZAT MSR } \\
(\mathrm{mm})\end{array}$ \\
\hline 1 & 3,78 & 3,6 & 5,5 & 5,16 \\
\hline 2 & 3,7 & 3,6 & 9,5 & 8,66 \\
\hline 3 & 2,99 & 3,2 & 8 & 8,54 \\
\hline 4 & 4,44 & 4,0 & 7 & 7,79 \\
\hline 5 & 3,34 & 3,2 & 4 & 4,16 \\
\hline 6 & 2,41 & 2,5 & 4,5 & 4,79 \\
\hline 7 & 2,2 & 2,2 & 6 & 5,04 \\
\hline 8 & 2,48 & 2,9 & 11 & 11,17 \\
\hline 9 & 1,84 & 2,2 & 2,5 & 3,66 \\
\hline 10 & 2,51 & 2,9 & 5 & 5,04 \\
\hline 11 & 3,46 & 3,5 & 7 & 8,29 \\
\hline 12 & 2,1 & 1,9 & 9 & 8,54 \\
\hline 13 & 1,68 & 1,6 & 7 & 6,29 \\
\hline 14 & 2,28 & 2,3 & 4,5 & 4,79 \\
\hline 15 & 2,23 & 2,0 & 7 & 7,67 \\
\hline 16 & 3,99 & 4,3 & 13,5 & 13,54 \\
\hline 17 & 3,34 & 3,8 & 15 & 14,66 \\
\hline 18 & 3,53 & 3,8 & 6,5 & 5,54 \\
\hline 19 & 1,71 & 2,2 & 6,5 & 7,16 \\
\hline 20 & 3,91 & 3,3 & 8,5 & 8,29 \\
\hline 21 & 3,17 & 3,13 & 10,5 & 11,04 \\
\hline 22 & 3,66 & 3,13 & 15,5 & 15,29 \\
\hline 23 & 2,23 & 2,13 & 5,5 & 5,04 \\
\hline 24 & 3,69 & 3,13 & 8 & 6,79 \\
\hline
\end{tabular}
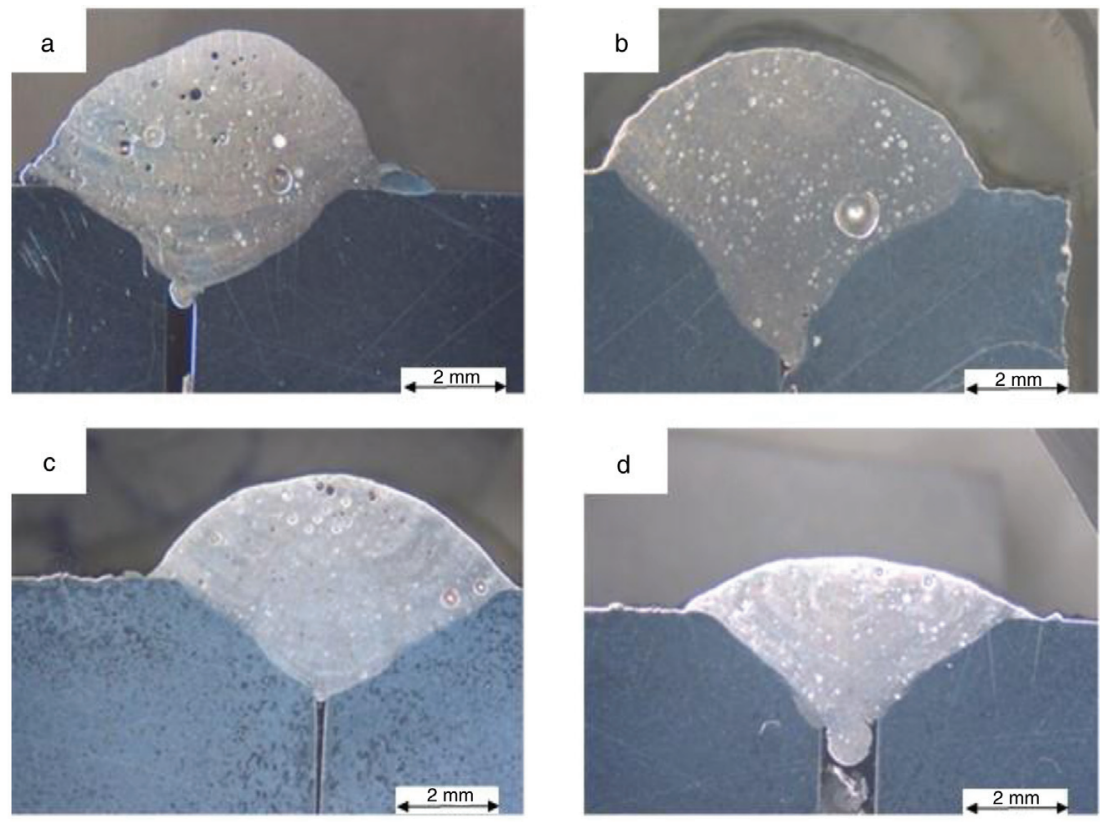

Figura 1. Detalle del cordón en el que se muestra la penetración para diferentes casos: a) experimento 7, b) experimento $11, \mathrm{c}$ ) experimento $15 \mathrm{y}$ d) experimento 20 . 

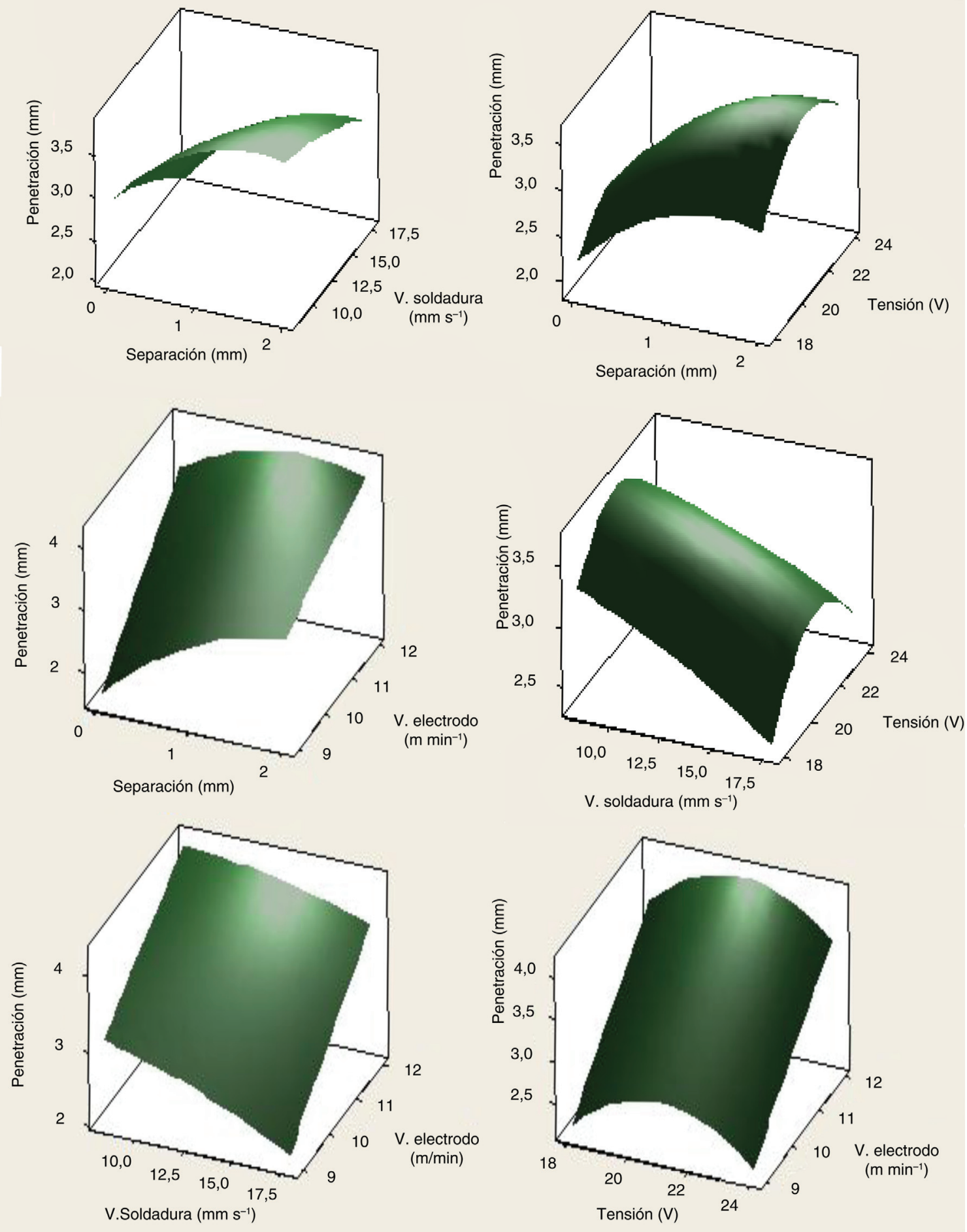

FIGURA 2. Gráficas de superficie de respuesta para la variable penetración.

la zona afectada térmicamente, lo que se justifica por la no influencia de este parámetro en la energía aportada en la soldadura. Se observa que la variable que más afecta a la zona afectada térmicamente es la velocidad de soldeo. Conforme la velocidad aumenta, disminuye la anchura de la zona afectada térmicamente. Este resultado es totalmente coherente con la influencia en la energía aportada en el soldeo que tiene el parámetro indicado. El resto de parámetros, tensión y velocidad de alimentación del 


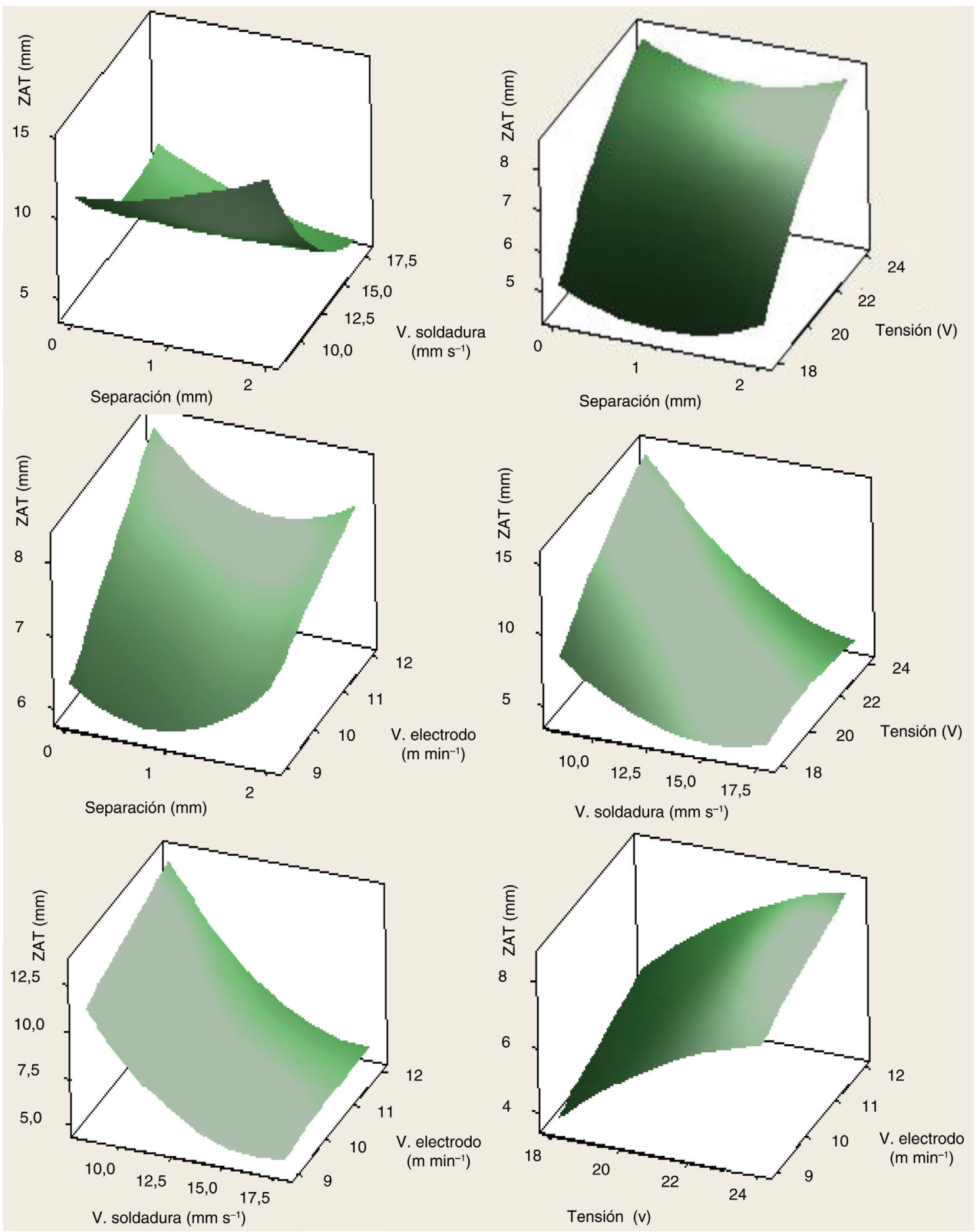

FIGURA 3. Gráficas de superficie de respuesta para la variable ZAT.

electrodo tienen una influencia secundaria con tendencia a aumentar la zona afectada térmicamente y coherente con el significado de tensión y velocidad del electrodo en relación a la energía aportada en el proceso. Dichas variables influyen de un modo similar en la penetración, a excepción de la separación de bordes que, lógicamente tiene una importancia elevada a diferencia de lo observado para la ZAT.

La optimización del proceso de soldeo teniendo en cuenta las variables indicadas debería perseguir como objetivo la maximización de la penetración con un valor mínimo de la ZAT. Para ello mediante 

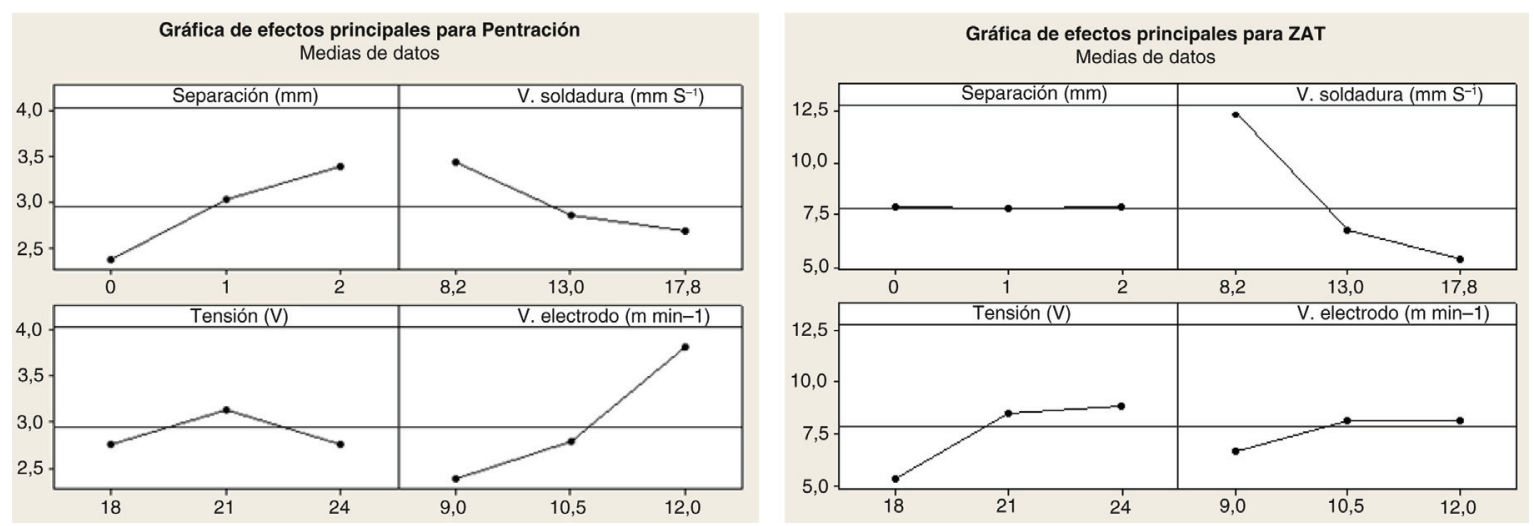

FIgURA 4. Gráficas de efectos principales para las variables penetración y ZAT.

el programa informático Minitab $16^{\circledR}$ se ha podido identificar la combinación de valores de variables de entrada dentro del rango de experimentación, que en conjunto optimizan cada una de las respuestas de forma individual así como el conjunto de las dos respuestas en el sentido indicado anteriormente. Planteando la maximización de la penetración y la minimización de la ZAT por separado, lo que sería un objetivo habitual en aplicaciones de tipo mecánico o estructural, se obtienen los resultados indicados en la Figura 5. Para ello se ha planteado un objetivo de optimización de las dos variables, pero eliminando las restricciones en una de ellas, respectivamente la penetración o la ZAT, para obtener el mínimo valor o el máximo de la variable restante. En dicha figura se observa también el valor de la variable penetración compatible con el valor mínimo de ZAT posible con los valores experimentados y viceversa, esto es, el valor compatible de la ZAT con la máxima penetración obtenible. También se establecen los valores de las variables tecnológicas que hacen posible la obtención de los valores de penetración y ZAT indicados.
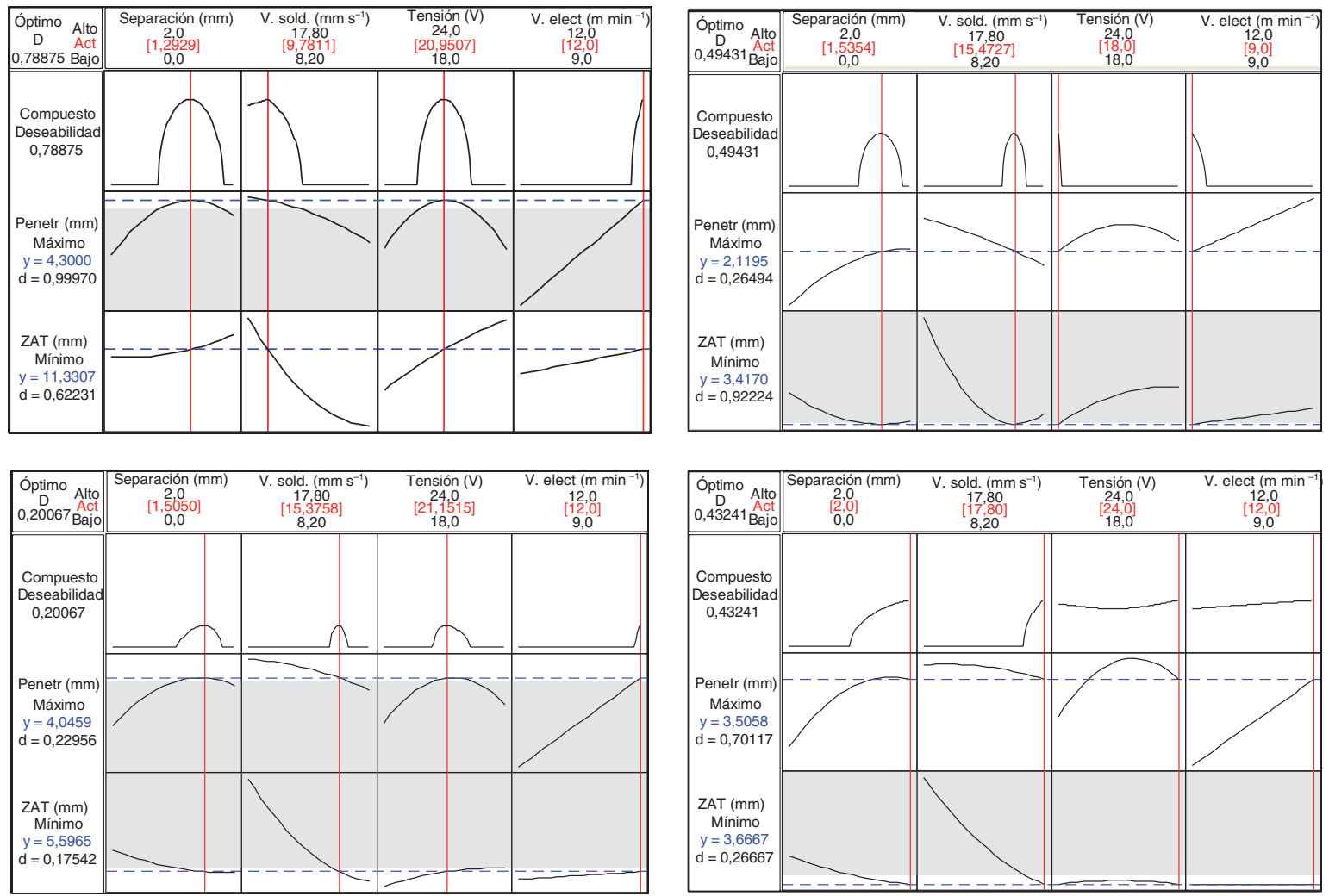

FIgURA 5. Optimización del proceso a partir del análisis MSR para diferentes valores objetivo de la penetración y de la ZAT. 
Una vez establecidos los valores máximo de penetración y mínimo de ZAT obtenibles dentro del rango de valores tecnológicos experimentados, se pueden establecer objetivos para cada una de las variables, obteniendo el mejor valor compatible de la otra variable mediante el método de optimización que nos da el análisis de la superficie de respuesta. Esta metodología permite conocer cómo afectan los valores tecnológicos al conjunto de las dos variables contempladas en esta investigación y optimizar el procedimiento de soldeo. Así, en la investigación efectuada se observa que el mayor valor de penetración es $4,3 \mathrm{~mm}$, que podría ser incluso suficiente en función del cálculo mecánico de la sección conforme al código de diseño que se adopte y que no tiene que involucrar necesariamente a todo el espesor de la pieza. Un ejemplo sencillo puede plantearse para el caso de la unión experimentada, cuyas piezas tienen un espesor de $8 \mathrm{~mm}$. Incluso aunque se fijara la penetración total como objetivo, éste se podría alcanzar mediante soldeo por ambas caras, con lo que una penetración de $4 \mathrm{~mm}$ podría ser suficiente. En la Figura 5 se muestra alguna situación más como el objetivo de rebajar la penetración a $3,5 \mathrm{~mm}$ minimizando de nuevo la ZAT.

En la Figura 6 se representan los valores tecnológicos que permiten optimizar los valores de la ZAT en función de la penetración. Se incluyen más valores que los mostrados en la Figura 5.

El análisis de la Figura 6 permite establecer que para el rango de valores experimentado de las variables tecnológicas involucradas, la ZAT se mantiene próxima al mínimo alcanzable hasta valores de penetración superiores a $3,5 \mathrm{~mm}$. Por encima de dicho valor la ZAT crece exponencialmente y pequeños cambios de los parámetros de soldeo con variaciones bajas de penetración conducen a valores muy elevados de la ZAT.

La velocidad del electrodo está directamente relacionada con la intensidad de corriente del arco en los procesos GMAW (Tlusty, 2000), estableciéndose que este parámetro es decisivo para regular la velocidad de fusión del material de aporte y, en consecuencia la ZAT. No obstante, se observa que la velocidad de soldeo es el parámetro más significativo en el control de la ejecución óptima del cordón. Como se observa en la Figura 6, en el rango de las altas penetraciones experimentadas ( 3,5 a $4,3 \mathrm{~mm}$ ) el parámetro que más modifica las condiciones del cordón es la velocidad de soldeo, por lo que resulta más eficiente actuar sobre dicho parámetro, especialmente si se quiere establecer un control simplificado de la operación.

\section{CONCLUSIONES}

- En el presente trabajo se ha establecido una metodología de control para soldadura GMAW basada en la metodología de la superficie de respuesta multiobjetivo sobre dos parámetros, penetración y longitud de la zona afectada térmicamente.

- La metodología experimental para la medición de ambas variables, basada en la preparación metalográfica de las uniones soldadas, ha permitido obtener funciones de respuesta más ajustadas a los experimentos que las establecidas por métodos de otros autores o incluso que las obtenidas para la penetración por los

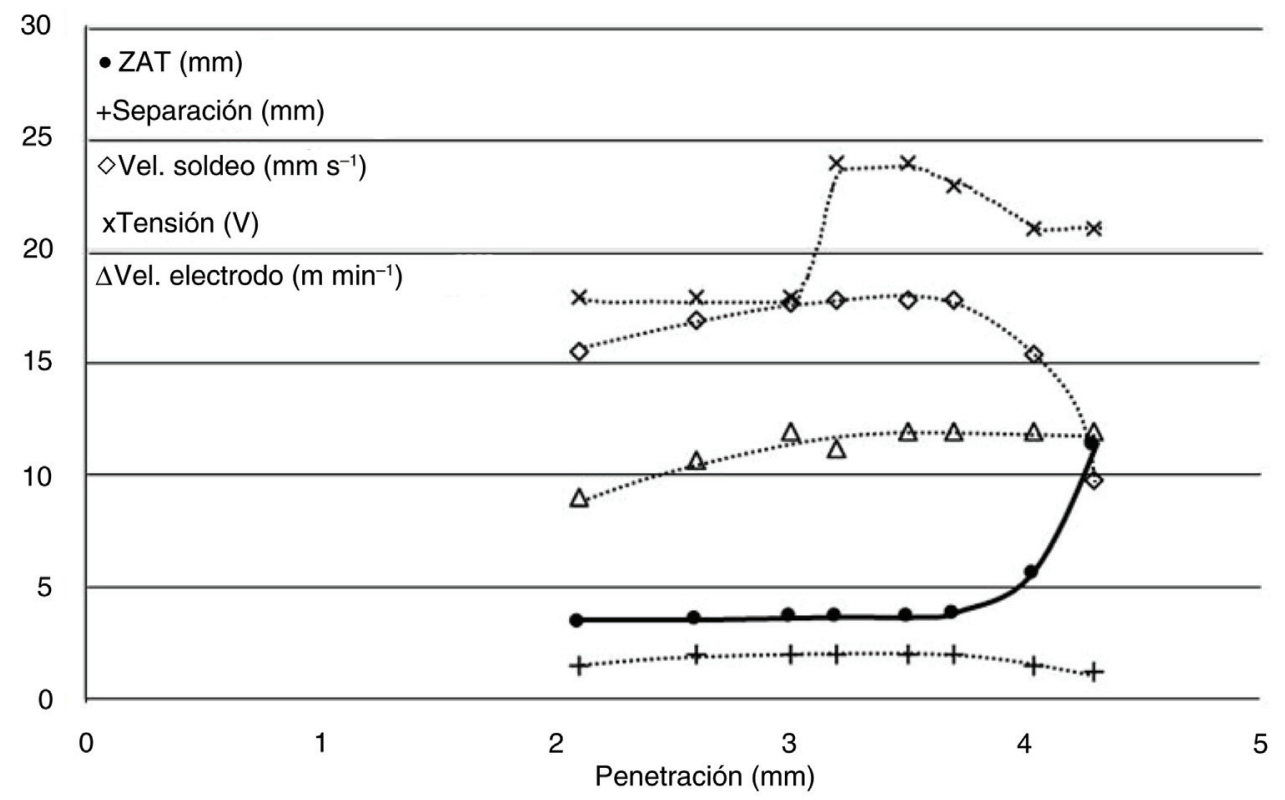

FIGURA 6. Representación de los valores tecnológicos que optimizan las variables penetración y ZAT para distintos valores objetivo de las variables. 
autores de este trabajo en un estudio anterior (Miguel et al., 2012). El error relativo medio que arroja el modelo es del 9\% para ambas variables, lo que supone un valor ajustado para este tipo de experimentos aplicados a procesos de soldeo por arco si se compara con los resultados de otros autores (Miguel et al., 2012; Kim et al., 2001; Kim et al., 2003).

- La cuantificación de la zona afectada térmicamente, junto con la penetración permite optimizar las variables tecnológicas del proceso estableciendo los criterios de control a emplear.

- Se ha determinado que la variable más significativa es la velocidad de soldeo. Pequeñas variaciones de la penetración en rangos próximos a los máximos alcanzables en condiciones de operatividad, suponen grandes variaciones de la ZAT, por lo que es aconsejable no establecer criterios de optimización definidos como maximización de la penetración, sino fijar valores operativos suficientes o diseñar secuencias de soldeo que permitan trabajar ligeramente por debajo del máximo valor. De este modo se consigue estabilizar en valores relativamente bajos la ZAT.

\section{AGRADECIMIENTOS}

Los autores desean agradecer al Instituto Politécnico de Cartagena la cesión de sus instalaciones para realizar la soldadura de las probetas empleadas en este trabajo. Particularmente agradecen a Francisco Segura y a Eugenio Abellán su colaboración en la realización de dichas soldaduras.

\section{REFERENCIAS}

Dorta, M., Vidal, J., Mateo, A., Fargas, G., Camejo, F. (2011). Modelos empíricos para la predicción de la geometría del cordón en soldaduras a tope de un acero inoxidable dúplex 2205. Dyna 78 (169), 206-215.

Karadeniz, E., Ozsarac, U., Yildiz, C. (2007). The effect of process parameters on penetration in gas metal arc welding processes. Mater. Desing 28 (2), 649-656. http://dx.doi. org/10.1016/j.matdes.2005.07.014

Kim, I.S., Son, J.S., Kim, I.G., Kim J.Y., Kim, O.S. (2003). A study on relationship between process variables and bead penetration for robotic $\mathrm{CO}_{2}$ arc welding. J. Mater. Process. Tech. 136 (1-3), 139-145. http://dx.doi.org/10.1016/ S0924-0136(02)01126-3.

Kim, I.S., Park, C. E., Jeong Y. J., Son, J. S. (2001). Development of an intelligent system for selection of the process variables in gas metal arc welding processes. Int. J. Adv. Manuf. Technol. 18, 98-102.

Martínez, A., Miguel, V., Coello, J., Navarro, A., Calatayud, A., Manjabacas, M.C. (2011). Influencia de la técnica de soldadura multipasada y de los tratamientos térmicos de precalentamiento y post-soldadura en el comportamiento de uniones GMAW de un acero microaleado HARDOX 400. Rev. Metal. 47 (1), 61-75. http://dx.doi.org/10.3989/ revmetalmadrid.1007.

Mc Glone, J.C., Chadwick, D.B. (1978). The submerged arc butt welding of mild steel. Part 2. The prediction of weld bead geometry from the procedure parameters. Welding Institute Report 80, 256-263.

Miguel, V., García-Conesa, E.J., Segura, F., Manjabacas M.C., Abellán, E. (2012). Optimización del proceso de soldadura GMAW de uniones a tope de la aleación AA 6063-T5 basada en la metodología de superficie de respuesta y en la geometría del cordón de soldadura. Rev. Metal. 48 (5), 333-350. http://dx.doi.org/10.3989/revmetalm.1169.

Miguel, V., Martínez, A., Manjabacas, M.C., Coello, J., Calatayud, A. (2009). Electrical Evaluation Of Welding Machines Based On The Arc Properties. Application To SMAW, GMAW And GTAW Processes. AIP Conference Proceedings 1181, New York, USA, pp. 170-179. http://dx.doi. org/10.1063/1.3273627.

Murray, P.E., Scotti, A. (1999). Depth of penetration in gas metal arc welding. Sci. Technol. Weld. Joi. 4 (2), 112-117. http://dx.doi.org/10.1179/136217199101537644.

Norma UNE-EN ISO 18273 (2005). Consumibles para el soldeo. Electrodos de alambre, alambres y varillas para el soldeo del aluminio y aleaciones de aluminio.

Norma UNE-EN ISO 6507-1(2006). Materiales metálicos. Ensayo de dureza Vickers. Parte 1: Método de ensayo.

Payares, M.C., De Barros, C., Muñoz, P., Cassier, Z. (1997). Modelo para la determinación del área del cordón en soldaduras a tope de aluminio 6063-T5. Rev. LatinAm. Metal. Mater. 17 (1), 5-12.

Tlusty, G. (2000). Manufacturing Processes and Equipment. Ed. Prentice-Hall, pp. 784-786 USA.

Wahab, M.A., Painter, M.J. (1997). Numerical models of metal arc welds using experimentally determined weld pool shapes as the representation of the welding heat source. Int. $J$. Press. Ves. Pip. 73 (2), 153-159. http://dx.doi.org/10.1016/ S0308-0161(97)00049-5. 